\title{
A predictive modeling study of the impact of chemical doping on the strength of a Ag/ ZnO interface
}

Cite as: J. Appl. Phys. 124, 235304 (2018); https://doi.org/10.1063/1.5051987

Submitted: 13 August 2018 . Accepted: 04 December 2018 . Published Online: 19 December 2018

Tianwei Wang (D), and Paul D. Bristowe (D)

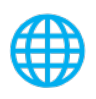

\section{ARTICLES YOU MAY BE INTERESTED IN}

Enhanced ultraviolet emission from self-assembled ZnO nanorods grown on graphene Journal of Applied Physics 124, 235302 (2018); https://doi.org/10.1063/1.5064395

Excitation intensity and thickness dependent emission mechanism from an ultrathin InAs layer in GaAs matrix

Journal of Applied Physics 124, 235303 (2018); https://doi.org/10.1063/1.5053412

Influence of deep levels on the electrical transport properties of CdZnTeSe detectors Journal of Applied Physics 124, 235702 (2018); https://doi.org/10.1063/1.5063850

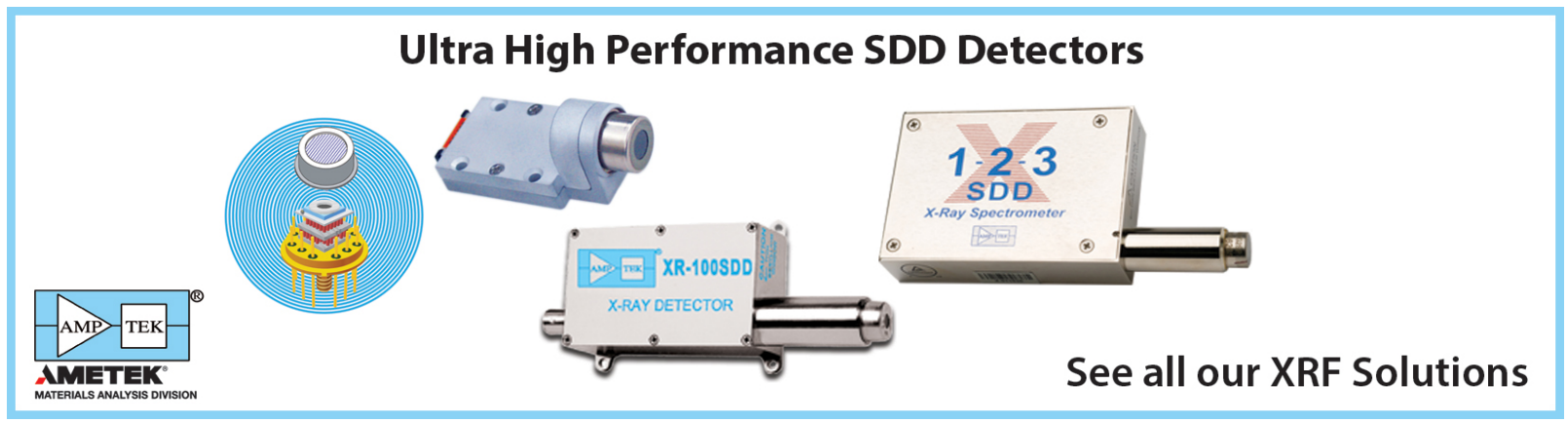




\title{
A predictive modeling study of the impact of chemical doping on the strength of a $\mathrm{Ag} / \mathrm{ZnO}$ interface
}

\author{
Tianwei Wang ${ }^{\text {a) }}$ and Paul D. Bristowe ${ }^{\text {b) }}$ \\ Department of Materials Science and Metallurgy, University of Cambridge, Cambridge CB3 OFS, \\ United Kingdom
}

(Received 13 August 2018; accepted 4 December 2018; published online 19 December 2018)

\begin{abstract}
Using first principles modeling, we predict how substitutional doping can influence the mechanical strength of a $\mathrm{Ag} / \mathrm{ZnO}$ interface commonly found in various multilayer thin-film systems. Replacing $\mathrm{Zn}$ with a monovalent dopant strengthens the interface while dopants with a valence greater than two weaken it. Isovalent dopants have little effect. The results are explained in terms of charge transfer and hybridization effects at the interface. Although monovalent dopants are mechanically preferred, they do not inhibit Ag inter-diffusion in $\mathrm{ZnO}$ and could possibly cause chemical degradation of the interface. It is suggested that other dopants, such as $\mathrm{Bi}$, avoid this issue by creating larger diffusion barriers while maintaining a relatively strong interface. The results indicate that complete control over the mechanics, kinetics, and chemistry of the interface requires a careful choice of dopant types and concentrations. Published by AIP Publishing. https://doi.org/10.1063/1.5051987
\end{abstract}

\section{INTRODUCTION}

Silver is often deposited on $\mathrm{ZnO}$ (doped or undoped) to enhance the conductivity as well as the low-emissivity of a conductive and transparent multilayer thin-film system. ${ }^{1,2}$ Such multilayer systems are widely used as solar coatings in the glass industry ${ }^{3}$ and as transparent electrodes in various electronics applications such as flat-panel displays, thin-film transistors, and solar cells. ${ }^{4-6}$ The observed orientation relationship is usually $\mathrm{Ag}(111) / \mathrm{ZnO}(0001)^{7-9}$ with the $\mathrm{ZnO}$ being oxygen-terminated (O-t) at the interface due to the stronger $\mathrm{Ag}-\mathrm{O}$ bonding and a relatively oxygen-rich environment during deposition. ${ }^{8}$

The current generation of such multilayer systems features an oxide/metal/oxide structure (e.g., $\mathrm{ZnO} / \mathrm{Ag} / \mathrm{ZnO}$ or a derivative thereof) and is often manufactured using magnetron sputtering. ${ }^{1,9}$ The [110] direction in the Ag (111) plane is found to align parallel to the [1100] direction in the $\mathrm{ZnO}$ (0001) plane resulting in a relatively small lattice mismatch of $2.6 \% .^{7}$ This mismatch together with differences in the thermal expansion coefficients of the materials involved and point defects produced during sputtering results in a residual compressive stress in the multilayer, which is typically $\sim 1.5$ $\mathrm{GPa}^{3}{ }^{3}$ The reduction of this stress is desirable since it may contribute to interfacial delamination. ${ }^{10}$ One way of reducing the stress is to adjust the lattice mismatch by doping $\mathrm{ZnO}$. For example, the addition of small amounts of $\mathrm{Al}$, which first principles calculations show substitute on $\mathrm{Zn}$ sites, ${ }^{11}$ lowers the stress to $\sim 0.8 \mathrm{GPa} .{ }^{12-14} \mathrm{~A}$ further benefit of doping with $\mathrm{Al}$ is that it inhibits the diffusion of $\mathrm{Ag}$ in $\mathrm{ZnO},{ }^{15}$ thus limiting the chemical degradation of the multilayer. The smaller crystal radius of $\mathrm{Al}(0.67 \AA)$ compared to $\mathrm{Zn}(0.74 \AA)^{16}$ provides a simple explanation for the stress relaxation. However,

\footnotetext{
${ }^{\text {a)} E m a i l: ~ t w 445 @ c a m . a c . u k . ~}$

b) Author to whom correspondence should be addressed: pdb1000@cam. ac.uk. Tel.: +44-(0)1223-334305.
}

further calculations on the strength of the $\mathrm{Ag}(111) /$ $\mathrm{AZO}(0001)$ interface $^{11}(\mathrm{AZO}=\mathrm{Al}$-doped $\mathrm{ZnO})$ show that the presence of $\mathrm{Al}$ actually weakens it despite the reduction in stress. This finding applies to an otherwise clean interface which does not contain adsorbed gases or water. Electronic structure analysis suggests that the weakening of the interface could be explained using a compensation mechanism in which interfacial oxygen atoms gain charge from $\mathrm{Al}$ and local $\mathrm{Ag}-\mathrm{O}$ bonds become weaker. ${ }^{11}$ Thus, the use of chemical doping to improve the mechanical properties of a $\mathrm{Ag} / \mathrm{ZnO}$ interface does not necessarily produce the intended result, and further theoretical studies are desirable. In this paper, we present a systematic first principles computational investigation into the strength of the $\mathrm{Ag}(111) / \mathrm{ZnO}(0001)$ interface doped with various additional elements including those commonly used in applications such as Sc and Sn. ${ }^{15,17,18}$ The formal valence of the dopant varies from 1 to 5 and the discussion focuses on the combined effects of charge transfer and orbital interactions. In this work, valence is defined as the number of valence electrons used in the pseudopotential calculations excluding any $d$-electrons that may be present. The implications of the results for the interdiffusion of $\mathrm{Ag}$ are also briefly discussed.

\section{COMPUTATIONAL METHOD}

The calculations were performed using the plane-wave based density functional theory (DFT) code VASP. ${ }^{19,20}$ The exchange-correlation functional was approximated using the generalised-gradient approximation (GGA) as parameterized by Perdew et al. ${ }^{21}$ and revised for solids (PBEsol). ${ }^{22}$ The electronic wave functions were described by the projector augmented wave (PAW) method. ${ }^{23,24}$ The chosen kinetic energy cutoff of $500 \mathrm{eV}$ was sufficient to produce a total energy convergence of less than $0.02 \mathrm{eV} / \mathrm{atom}$. The electronic structure calculations were carried out with a Gaussian smearing width of $0.05 \mathrm{eV}$ and a convergence tolerance of $10^{-8} \mathrm{eV}$. Gamma-centered $5 \times 5 \times 2 \mathrm{k}$-point meshes were 
TABLE I. Calculated formation energies $\Delta E_{f}(\mathrm{eV})$ and chemical potentials $\mu(\mathrm{eV})$ of various dopants in $\mathrm{ZnO}$ substituted on a $\mathrm{Zn}$ site, an $\mathrm{O}$ site, or occupying an octahedral interstitial.

\begin{tabular}{lrrrr}
\hline \hline & $\mu_{\text {dopant }}$ & $\Delta E_{f, Z n}$ & $\Delta E_{f, o}$ & \multicolumn{1}{c}{$\Delta E_{f, i}$} \\
\hline $\mathrm{Li}$ & -4.794 & 0.239 & 6.107 & 2.226 \\
$\mathrm{Na}$ & -3.266 & 0.271 & 7.259 & 2.865 \\
$\mathrm{Cu}$ & -5.608 & 0.956 & 7.143 & 3.985 \\
$\mathrm{Ag}$ & -3.425 & 1.059 & 5.914 & 3.741 \\
$\mathrm{Au}$ & -3.924 & 1.663 & 5.141 & 3.959 \\
$\mathrm{Mg}$ & -7.386 & 0.179 & 10.502 & 5.887 \\
$\mathrm{Ca}$ & -8.284 & 0.512 & 11.072 & 6.311 \\
$\mathrm{~B}$ & -13.175 & 2.021 & 13.981 & 8.145 \\
$\mathrm{Al}$ & -11.976 & 1.560 & 14.415 & 9.251 \\
$\mathrm{Sc}$ & -15.788 & 2.044 & 13.544 & 8.991 \\
$\mathrm{Ga}$ & -8.284 & 1.178 & 11.869 & 7.888 \\
$\mathrm{In}$ & -7.220 & 1.695 & 11.475 & 7.451 \\
$\mathrm{Tl}$ & -3.519 & 0.272 & 8.047 & 5.155 \\
$\mathrm{Si}$ & -14.414 & 3.635 & 15.810 & 11.057 \\
$\mathrm{Ge}$ & -10.325 & 3.179 & 12.129 & 9.248 \\
$\mathrm{Sn}$ & -9.580 & 3.441 & 12.750 & 9.744 \\
$\mathrm{~Pb}$ & -6.309 & 0.966 & 9.972 & 7.236 \\
$\mathrm{P}$ & -12.962 & 5.346 & 12.091 & 10.639 \\
$\mathrm{Bi}$ & -7.560 & 2.333 & 10.232 & 8.715 \\
\hline \hline
\end{tabular}

used for the interface calculations and a slightly reduced density of $0.03 \AA^{-1}$ was used for all other associated bulk and defect calculations. The atomic positions were relaxed using the conjugate gradient $(\mathrm{CG})$ method using a tolerance of $0.01 \mathrm{eV} / \AA$ for all force residuals. Charge transfer effects were determined by plotting charge density difference maps. Analysis on the interfacial bond strengths was performed using the projected Crystal Orbital Hamilton Population (pCOHP) $)^{25,26}$ method as implemented in the LOBSTER code. $^{27,28}$ The interfacial strengths were determined by calculating the ideal work of separation ${ }^{2,13}$ and atomic structures were visualised using VESTA. ${ }^{29}$

\section{A. Setup for the formation energies}

Before proceeding to the interface calculations, it is necessary to confirm the preferred incorporation mechanism in the bulk for each dopant considered. Following previous calculations on $\mathrm{Ag}$ diffusion in doped $\mathrm{ZnO},{ }^{15}$ the formation energies of 19 dopants were determined and the results are summarised in Table I. An orthorhombic supercell containing 96 atoms was employed based on the primitive hexagonal cell of $\mathrm{ZnO}$. Both the supercell dimensions and the fractional coordinates of the atoms were relaxed to obtain the formation energies. The lattice parameters of pure $\mathrm{ZnO}$ obtained using the PBEsol functional $(a=b=3.236 \AA$ and $c=5.230 \AA)$ agreed well with the experimental values of $a_{0}=b_{0}=3.250 \AA$ and $c_{0}=5.207 \AA .^{30} \mathrm{Zn}$ and $\mathrm{O}$ substitutions were considered together with an octahedral interstitial. Oxygen-rich conditions were assumed since these were closest to the deposition environment at the $\mathrm{ZnO}$ surface, and this resulted in oxygen and zinc chemical potentials of $\mu_{\mathrm{O}}=-5.126 \mathrm{eV}$ and $\mu_{\mathrm{Zn}}=-4.577 \mathrm{eV}$, respectively. The chemical potentials were obtained as described in the work by Lin and Bristowe. ${ }^{11}$ It is seen that all dopants favor $\mathrm{Zn}$ substitution under these conditions, which agrees well with other computational work. ${ }^{31}$ Therefore, when considering chemical doping at the interface, only substitution on the $\mathrm{Zn}$ site was considered.

\section{B. Setup for the interface calculations}

In addition to determining the incorporation mechanism, it is important to verify that the dopants are likely to be

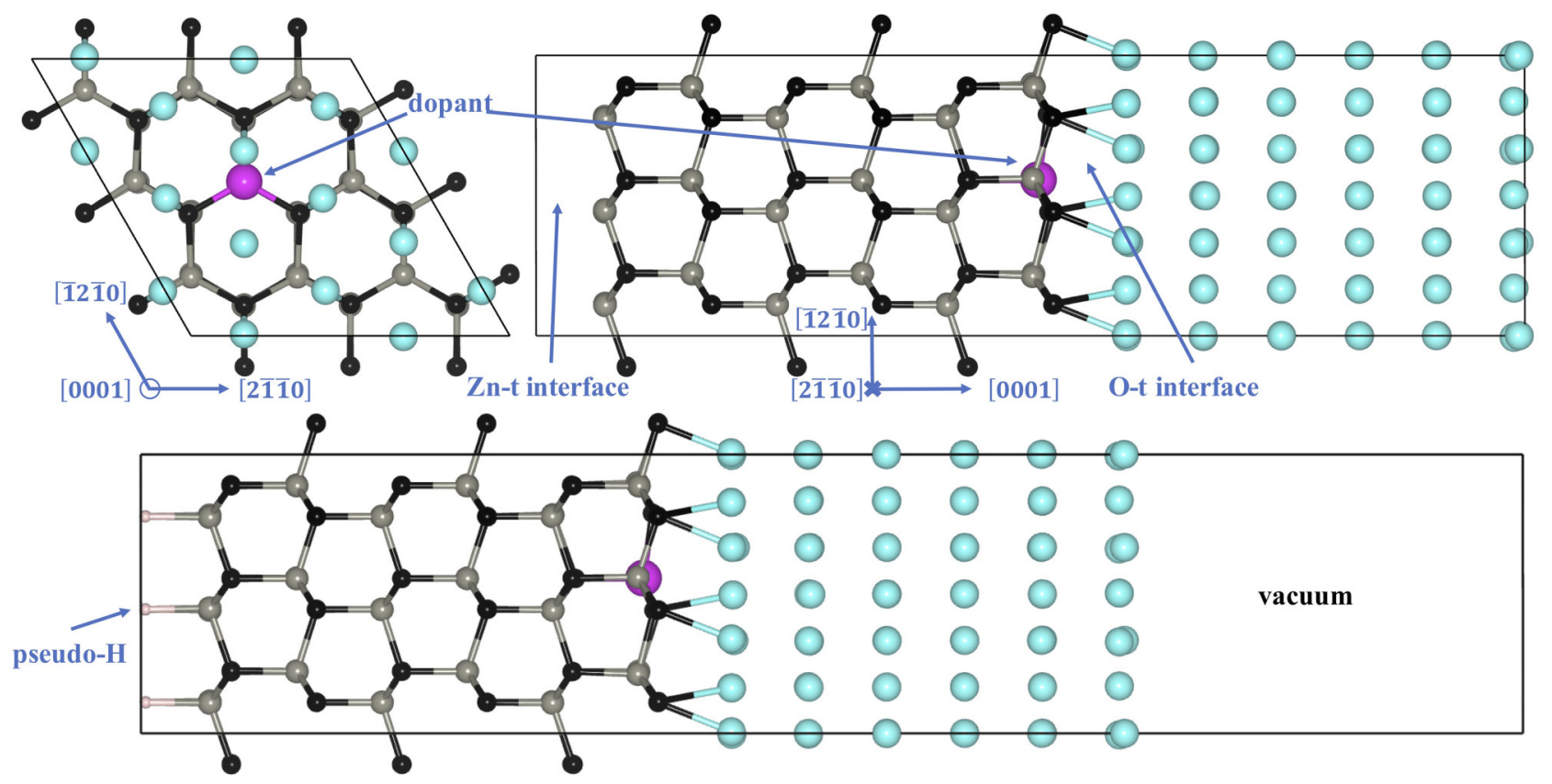

FIG. 1. Plan (upper-left) and side (upper-right) views of the $\mathrm{Ag}(111) / \mathrm{ZnO}(0001)$ supercell with two interfaces: a zinc-terminated (Zn-t) interface and an oxygen-terminated (O-t) interface. $\mathrm{Zn}, \mathrm{O}$, and $\mathrm{Ag}$ atoms are coloured gray, black, and cyan, respectively. A dopant atom is coloured purple. Only one layer of $\mathrm{Ag}$ atoms adjacent to $\mathrm{ZnO}$ is shown in the plan view. The lower view shows the extended supercell with a vacuum ( $>10 \AA)$ used to calculate the work of separation. 

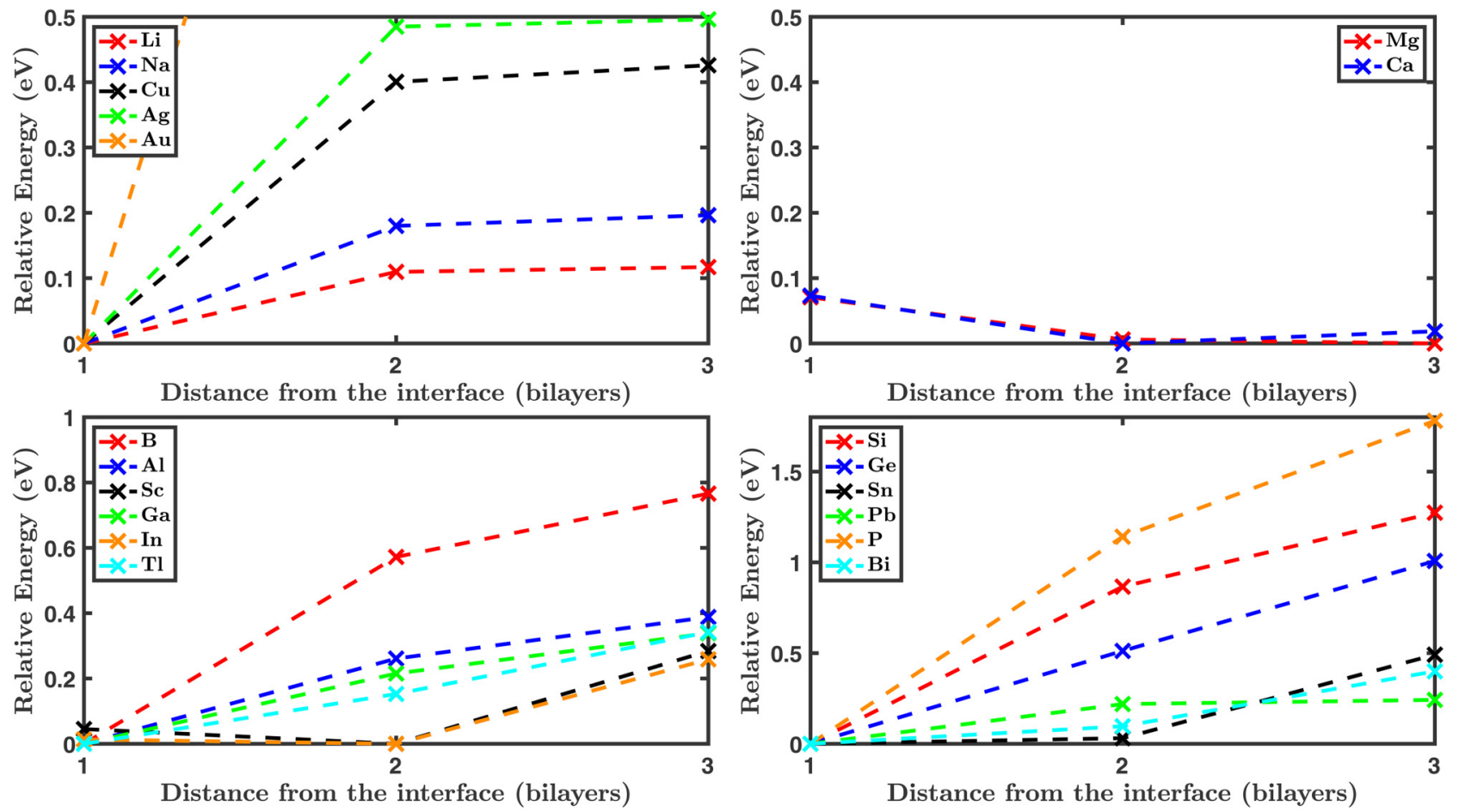

FIG. 2. The relative energy (eV) of each doped system as a function of the dopant distance (number of $\mathrm{ZnO}$ bilayers) from the O-t Ag/ZnO interface. The energy is relative to the lowest total energy of the supercell in each case.

present at the $\mathrm{Ag} / \mathrm{ZnO}$ interface, i.e., there is a driving force for segregation. To demonstrate this, hexagonal interfacial supercells $(a=b=9.750 \AA$ and $c=30.00 \AA)$ were constructed by combining primitive cells of $\mathrm{ZnO}$ and $\mathrm{Ag}\left(a_{0}=2.89 \AA^{32}\right)$ using the observed epitaxial orientation relationship. Periodic boundary conditions were applied and two interfaces were created, one oxygen-terminated and the other zinc-terminated as shown in the top part of Fig. 1. The $\mathrm{ZnO}$ slab consisted of 6 (0001) bilayers each containing 18 atoms. The Ag slab consisted of 6 (111) layers each containing 12 atoms and to obtain lattice matching with the $\mathrm{ZnO}$, each layer was biaxially compressed by $2.6 \%$. During optimisation, the supercell dimensions and all atomic positions in the supercell were allowed to relax without constraint. As a result, the interfacial crosssectional areas and interface spacings were simultaneously optimised thereby minimising the stress. A dopant atom was substituted into the $\mathrm{ZnO}$ slab at various distances from the O- $\mathrm{t}$ $\mathrm{Ag} / \mathrm{ZnO}$ interface. The relative energy of each doped system as a function of the dopant distance from the interface is shown in Fig. 2. It is seen that all dopants (except for $\mathrm{Mg}, \mathrm{Ca}$, and $\mathrm{Sc}$ ) tend to segregate to the interface, some more strongly than others. Although $\mathrm{Mg}, \mathrm{Ca}$, and $\mathrm{Sc}$ show no clear driving force to segregate immediately adjacent to the interface, it can be assumed that some fraction of these elements will lie near the interface. In the work of separation calculations that follow, each dopant was substituted into the $\mathrm{Zn}$ layer right next to the O-t interface as shown in Fig. 1.

\section{RESULTS AND DISCUSSION}

\section{A. Ideal work of separation}

To determine the strength of each doped $\mathrm{Ag} / \mathrm{ZnO}$ interface, the supercells described and relaxed in Sec. II B were then extended along $c$ using a vacuum ( $>10 \AA$ ) to eliminate the Zn-t interface as shown in the lower part of Fig. 1. The $\mathrm{Zn}$ - $\mathrm{t}$ free surface of $\mathrm{ZnO}$ was passivated by pseudo- $\mathrm{H}$ atoms having a charge of $1 / 2$ electron. Such passivation has been shown to be sufficiently effective by checking the electrostatic potential along the [0001] direction of $\mathrm{ZnO}$

TABLE II. Valence of the dopants considered in this work (values in brackets include the $d$-electrons) together with the interfacial area $A$ and the ideal work of separation ( $\mathrm{Zn}$ indicates the undoped interface which has a value of $2.006 \mathrm{~J} / \mathrm{m}^{2}$ ). The total calculated interfacial bond strengths (-iCOHP) are also given as displayed in Fig. 4.

\begin{tabular}{lcccc}
\hline \hline & Valence & $A\left(\AA^{2}\right)$ & $W_{\text {sea }}^{\text {ideal }}\left(\mathrm{J} / \mathrm{m}^{2}\right)$ & $-\mathrm{iCOHP}(\mathrm{eV})$ \\
\hline $\mathrm{Li}$ & 1 & 83.07 & 2.419 & 46.42 \\
$\mathrm{Na}$ & 1 & 83.18 & 2.395 & 46.06 \\
$\mathrm{Cu}$ & $1(11)$ & 83.25 & 2.143 & 46.38 \\
$\mathrm{Ag}$ & $1(11)$ & 83.35 & 2.175 & 46.46 \\
$\mathrm{Au}$ & $1(11)$ & 83.42 & 2.195 & 47.00 \\
$\mathrm{Mg}$ & 2 & 83.07 & 2.052 & 41.91 \\
$\mathrm{Ca}$ & 2 & 83.20 & 2.041 & 43.02 \\
$\mathrm{Zn}$ & 2 & 83.05 & 2.006 & 42.01 \\
$\mathrm{~B}$ & 3 & 82.59 & 1.644 & 38.74 \\
$\mathrm{Al}$ & 3 & 82.89 & 1.629 & 37.50 \\
$\mathrm{Sc}$ & 3 & 83.09 & 1.640 & 38.76 \\
$\mathrm{Ga}$ & $3(13)$ & 82.96 & 1.633 & 37.35 \\
$\mathrm{In}$ & $3(13)$ & 83.19 & 1.643 & 37.92 \\
$\mathrm{Tl}$ & $3(13)$ & 83.30 & 1.680 & 39.87 \\
$\mathrm{Si}$ & 4 & 82.76 & 1.258 & 33.52 \\
$\mathrm{Ge}$ & $4(14)$ & 82.88 & 1.258 & 33.29 \\
$\mathrm{Sn}$ & $4(14)$ & 83.17 & 1.306 & 35.24 \\
$\mathrm{~Pb}$ & $4(14)$ & 83.30 & 1.700 & 42.05 \\
$\mathrm{P}$ & 5 & 82.74 & 0.928 & 29.64 \\
$\mathrm{Bi}$ & $5(15)$ & 83.27 & 1.485 & 38.76 \\
\hline
\end{tabular}


(see Fig. S1 in the supplementary material). The supercell size was biaxially fixed using the cell dimensions from the previous optimisation. The total energy of this extended supercell $E_{\text {Bicrystal }}^{\mathrm{Ag} / \mathrm{ZnO}}$ was determined without further relaxation. The ideal work of separation $W_{\text {sep }}^{\text {ideal }}$ is defined as the reversible work needed to cleave the relaxed interface if subsequent surface diffusion and reconstructions are neglected,

$$
W_{\text {sea }}^{\text {ideal }}=\left(E_{\text {Slab }}^{\mathrm{ZnO}}+E_{\text {Slab }}^{\mathrm{Ag}}-E_{\text {Bicrystal }}^{\mathrm{Ag} / \mathrm{ZnO}}\right) / A .
$$

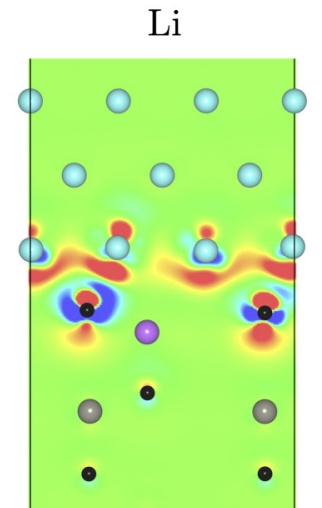

$\mathrm{Mg}$

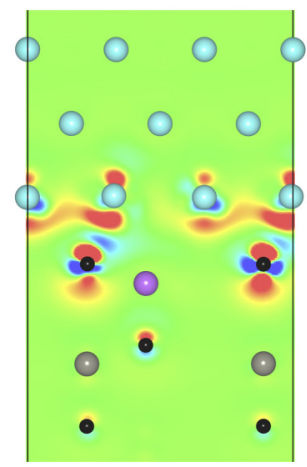

$\mathrm{Sc}$

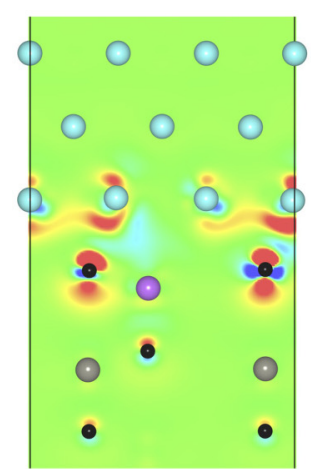

$\mathrm{Ge}$

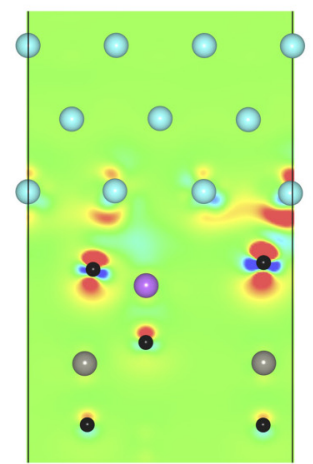

$\mathrm{Na}$

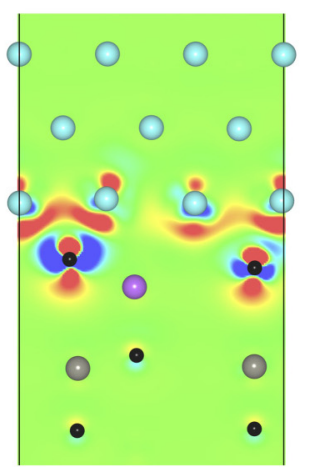

$\mathrm{Ca}$

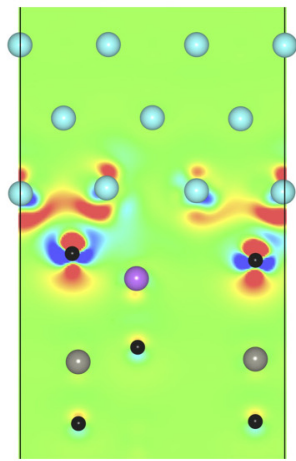

$\mathrm{Ga}$

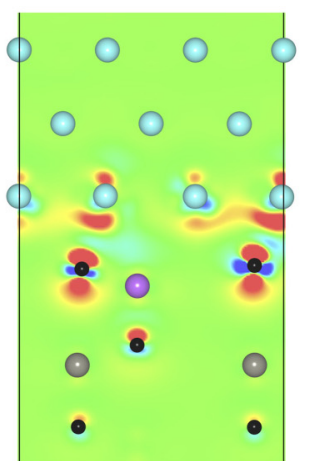

$\mathrm{Sn}$

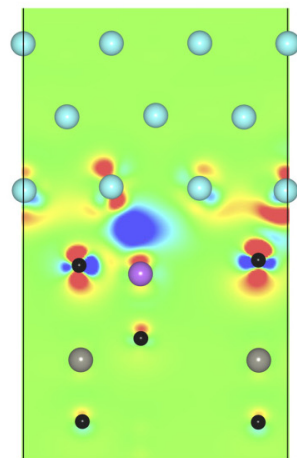

$\mathrm{Cu}$

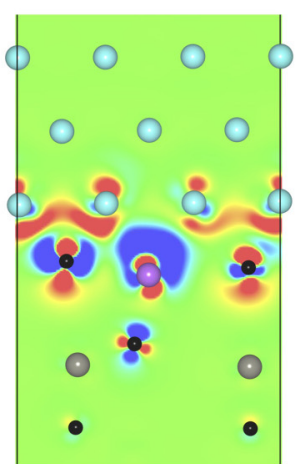

$\mathrm{Zn}$

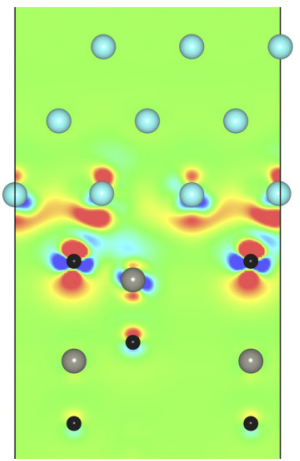

In

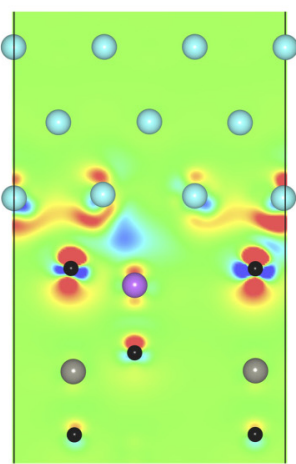

$\mathrm{Pb}$

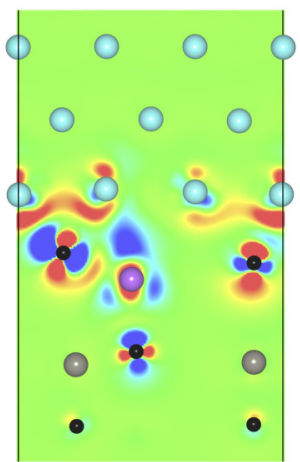

Ag

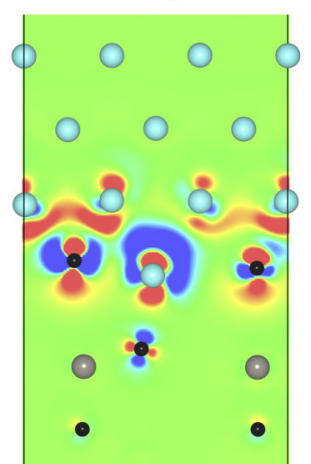

B

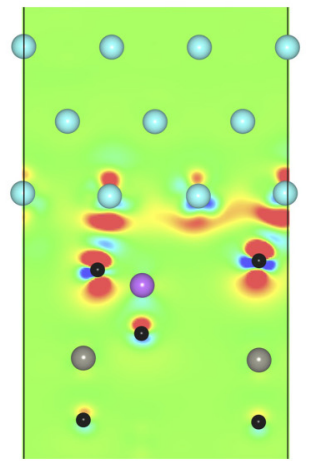

$\mathrm{Tl}$

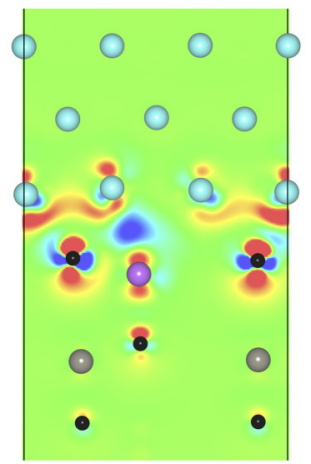

P

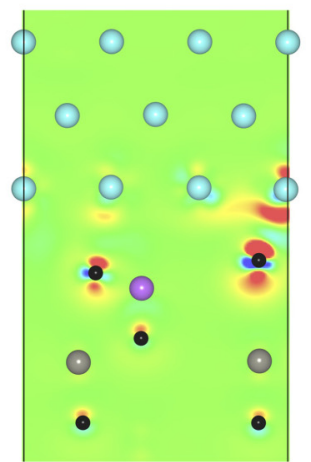

$\mathrm{Au}$

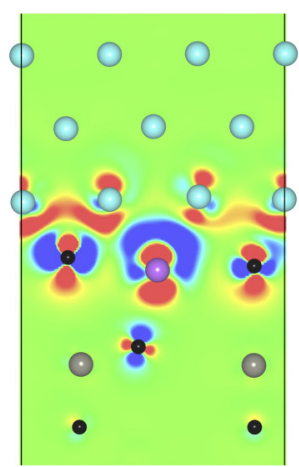

$\mathrm{Al}$

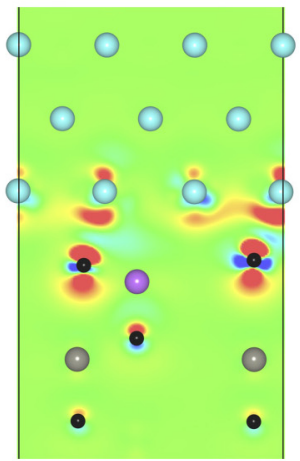

$\mathrm{Si}$

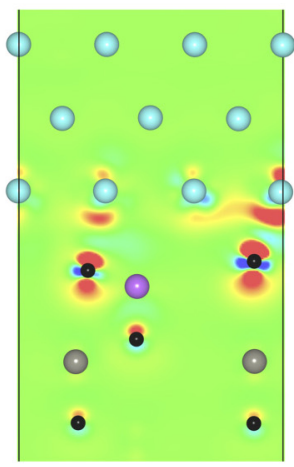

Bi

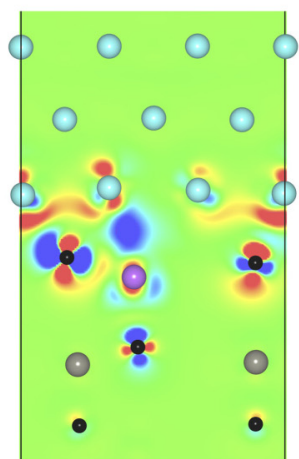

FIG. 3. The difference in charge density between the interface model and the sum of the two separated slabs, viewed along [2110] in ZnO. A slice is taken through the supercell which passes through the dopant, Zn, and O atoms. Using an RGB scale, red and blue regions represent charge density gain and loss, respectively, with a density range of -0.006 to $0.0051 / \mathrm{bohr}^{3}$. 
The term $E_{\text {slab }}$ is the total energy of each individual slab and $A$ is the interfacial cross-sectional area. The individual slab energies were obtained by replacing one of the relaxed slabs in the supercell with vacuum and allowing no further relaxation. The results are given in Table II, which also lists the formal valence of each dopant (i.e., the number of valence electrons treated explicitly by the pseudopotential) together with the optimised values of the cross-sectional area. It is seen that $W_{\text {sep }}^{\text {ideal }}$ decreases as the dopant valence increases, i.e., relative to the undoped interface, monovalent ions strengthen it while trivalent, tetravalent, and pentavalent ions weaken it. However, there is no obvious relationship with the interface area.

The relationship between $W_{\text {sep }}^{\text {ideal }}$ and dopant valence can be understood in terms of a simple charge transfer mechanism. For example, a dopant with a valence greater than that of $\mathrm{Zn}$ will donate a fraction of the excess charge to its $\mathrm{O}$ neighbours and, in particular, to the $\mathrm{O}$ atom immediately adjacent to the interface (which is O-terminated). The increased charge on this $\mathrm{O}$ atom suppresses charge transfer from its neighbouring $\mathrm{Ag}$ atom across the interface resulting in a weaker $\mathrm{Ag}-\mathrm{O}$ bond. The opposite effect occurs when the dopant has a valence less than $\mathrm{Zn}$ leading to a reduction in charge on the interfacial $\mathrm{O}$ atoms and a strengthening of the $\mathrm{Ag}-\mathrm{O}$ bonds. Evidence for this model can be seen in Fig. 3 which shows charge density difference maps near the interface when it is doped with different elements. For example, the red regions at the interface coinciding with $\mathrm{Ag}-\mathrm{O}$ bonds generally reduce in size as the valence of the dopant increases (red represents charge density gain relative to the separated slabs). There are exceptions to this such as $\mathrm{Tl}, \mathrm{Pb}$, and $\mathrm{Bi}$, and these are discussed further below. However, the overall trend is a progressive weakening of the interfacial bond strengths across the series.

\section{B. Interfacial bond strength}

Since the charge density difference is only a qualitative measure of the interfacial bonding, further quantitative analysis of the bond strengths was performed. The pCOHP method was used in which the plane-wave basis sets were analytically transferred to an orbital basis. ${ }^{26,27}$ Integration of the pCOHP up to the Fermi energy $\epsilon_{F}$ represents the contribution of the interaction between two atoms to the band energy (bonding or anti-bonding) and is indicative of the bond strength. The integrated pCOHP (iCOHP) is given by

$$
\mathrm{iCOHP}=\int^{\epsilon_{F}} \operatorname{pCOHP}(E) \mathrm{d} E .
$$

Figure 4 compares the value of -iCOHP, summed over all atom pairs with a distance less than $5 \AA$ across the interface, with the calculated ideal work of separation for each doped system. The negative sign for iCOHP indicates an overall bonding interaction. It is seen that -iCOHP generally follows the ideal work of separation and decreases with increasing dopant valence.

\section{Discussion on the interfacial bonds}

Although the -iCOHP generally matches the ideal work of separation, deviations can be seen across different dopants

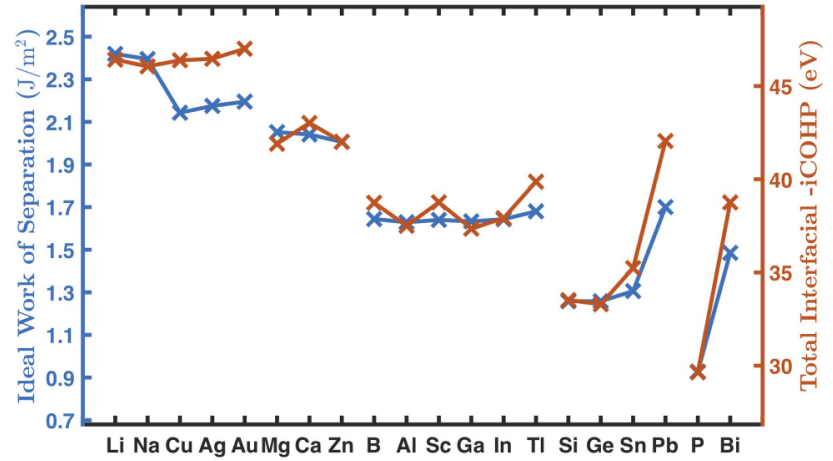

FIG. 4. Ideal work of separation $W_{\text {sep }}^{\text {ideal }}$ and interfacial bond strength $(-\mathrm{iCOHP})$ as a function of dopant. The two sets of results are superimposed by making the undoped $(\mathrm{Zn})$ values coincide. $W_{\text {sea }}^{\text {ideal }}$ for the undoped interface is $2.006 \mathrm{~J} / \mathrm{m}^{2}$.

(e.g., $\mathrm{Cu}, \mathrm{Ag}, \mathrm{Au}, \mathrm{Tl}, \mathrm{Sn}, \mathrm{Pb}$, and $\mathrm{Bi}$ ). It should be emphasized that the COHP method and the ideal work of separation are mechanically and chemically different methods of assessing the interfacial adhesion and their results may not necessarily match. Nevertheless, it is important to understand the origin of any differences. One significant observation is that for the list of special dopants given above, there are noticeable regions of charge density loss (shown in blue in Fig. 3) adjacent to the dopant and pointing toward the Ag slab. There are corresponding regions of charge density gain (shown in red) away from the $\mathrm{Ag}$ slab. This indicates the presence of an additional interaction between the dopant and the $\mathrm{Ag}$ slab in these cases. More quantitative evidence is given in Table III which shows values of -iCOHP between

TABLE III. The -iCOHP bond strengths, $f_{1}$ and $f_{2}$, between the dopant and its two closest $\mathrm{Ag}$ atoms in the slab with bond lengths $l_{1}$ and $l_{2}$. The mean interfacial spacing $d$ is included for comparison. Horizontal dotted lines separate the formal valence groups.

\begin{tabular}{|c|c|c|c|c|c|}
\hline & $f_{1}(\mathrm{eV})$ & $f_{2}(\mathrm{eV})$ & $l_{1}(\AA)$ & $l_{2}(\AA)$ & $d(\AA)$ \\
\hline $\mathrm{Li}$ & 0.738 & 0.375 & 2.871 & 3.227 & 2.074 \\
\hline $\mathrm{Na}$ & 0.647 & 0.352 & 2.963 & 3.313 & 2.072 \\
\hline $\mathbf{C u}$ & 1.672 & 1.426 & 2.678 & 2.746 & 2.101 \\
\hline Ag & 1.626 & 1.391 & 2.752 & 2.837 & 2.097 \\
\hline $\mathbf{A u}$ & 2.044 & 2.089 & 2.749 & 2.715 & 2.123 \\
\hline $\mathrm{Mg}$ & 0.832 & 0.408 & 2.983 & 3.327 & 2.156 \\
\hline $\mathrm{Ca}$ & 0.822 & 0.345 & 3.057 & 3.413 & 2.151 \\
\hline $\mathrm{Zn}$ & 1.014 & 0.423 & 2.931 & 3.306 & 2.153 \\
\hline B & 0.297 & 0.116 & 3.041 & 3.382 & 2.259 \\
\hline $\mathrm{Al}$ & 0.731 & 0.309 & 3.070 & 3.419 & 2.254 \\
\hline $\mathrm{Sc}$ & 1.024 & 0.408 & 3.074 & 3.416 & 2.244 \\
\hline $\mathrm{Ga}$ & 0.808 & 0.326 & 3.062 & 3.416 & 2.246 \\
\hline In & 1.253 & 0.522 & 3.004 & 3.366 & 2.225 \\
\hline Tl & 2.053 & 0.663 & 2.908 & 3.362 & 2.198 \\
\hline $\mathrm{Si}$ & 0.466 & 0.179 & 3.223 & 3.561 & 2.298 \\
\hline $\mathrm{Ge}$ & 0.609 & 0.232 & 3.194 & 3.538 & 2.334 \\
\hline Sn & 1.762 & 0.638 & 2.945 & 3.341 & 2.352 \\
\hline $\mathbf{P b}$ & 2.037 & 0.568 & 3.009 & 3.474 & 2.162 \\
\hline $\mathrm{P}$ & 0.254 & 0.095 & 3.385 & 3.711 & 2.452 \\
\hline $\mathbf{B i}$ & 2.274 & 0.585 & 2.980 & 3.473 & 2.236 \\
\hline
\end{tabular}




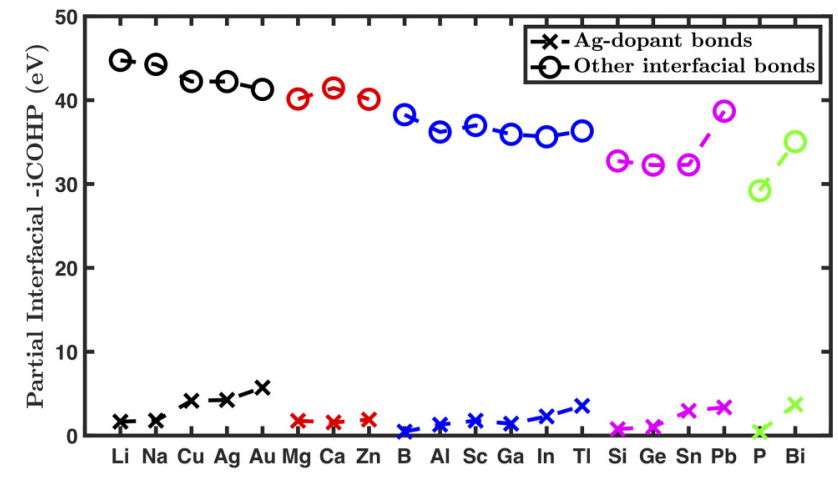

FIG. 5. Partial interfacial bond strength (-iCOHP) due to Ag-dopant bonds (crosses) and all other interfacial bonds (circles) as a function of dopant. Colours indicate different formal valence groups.

the dopant and the two closest Ag atoms in the slab, together with the corresponding bond distances. Values in bold are different from others in the same valence group in that the bond strengths are larger and the bond distances smaller. This is despite the fact that the mean interfacial spacing is sometimes larger in these cases.

Further evidence can be observed in Fig. 5, in which the total -iCOHP bond strength in Fig. 4 is separated into two groups: Ag-dopant bonds and all other interfacial bonds (Ag-O and $\mathrm{Ag}-\mathrm{Zn}$ ). The Ag-dopant bonds associated with a special dopant are much stronger than other dopants with the same valence, while the sum of the $\mathrm{Ag}-\mathrm{Zn}$ and $\mathrm{Ag}-\mathrm{O}$ bond strengths generally follows $W_{\text {sep }}^{\text {ideal }}$ and the charge transfer mechanism. Although indium also exhibits relatively strong Ag-dopant bonds, weak $\mathrm{Ag}-\mathrm{Zn}$ and $\mathrm{Ag}-\mathrm{O}$ bonds as a result of charge transfer reduce the total interface bond strength, and no obvious deviation is observed for this dopant.

To understand the Ag-dopant bonds better, orbitalwise contributions to these interactions have been calculated. Figure 6 shows that major contributions from the dopant (in this case either $\mathrm{Bi}$ or $\mathrm{P}$ ) are from the $s$ - and $p$-orbitals, while the $d$-orbital has little effect. This is evident from both the density of states and the iCOHP results. For both dopants, hybridization occurs just below the Fermi level between occupied $s$ - and $p$-orbitals of the dopant and the occupied orbitals of Ag. However, the effect is stronger for $\mathrm{Bi}$ because its $d$-orbital, although low-lying, shifts the $s$ - and $p$-orbitals upwards allowing more overlap with the Ag orbitals.

The regions of charge density loss between the special dopants and the Ag slabs seen in Fig. 3 further suggest that these elements provide additional charge at the $\mathrm{O}-\mathrm{t} \mathrm{ZnO}$ surface after separation of the interface. These additional charges partially passivate the polar surface and are energetically favored, which may result in an underestimation of the slab energy compared to the other dopants and subsequently produce the underestimation of $W_{\text {sep }}^{\text {ideal }}$ seen in Fig. 4.

In Sec. III A, it was noted that $W_{\text {sep }}^{\text {ideal }}$ generally decreases as the dopant valence increases with the possible exception of $\mathrm{Tl}, \mathrm{Pb}$, and $\mathrm{Bi}$. This can be explained by the relatively inactive $6 s$ electrons of these elements, which limits charge transfer to the neighbouring $\mathrm{O}$ atoms and therefore strengthens the Ag-O bonds. Evidence for this can be found in the density of states of these elements (see Fig. S2 in the supplementary material) which shows that the $6 s$ states of these elements are mostly located below the Fermi level. This produces interfacial bonds of comparable strength to the pure $\mathrm{ZnO}$ interface and are stronger than interfaces doped with most trivalent dopants. The large crystal radius of each of these three dopants (Tl: $1.02 \AA, \mathrm{Pb}: 0.92 \AA, \mathrm{Bi}: 1.16 \AA)^{16}$ leads to an increase in interfacial area as seen in Table II. It also means that they can be used to inhibit $\mathrm{Ag}$ diffusion in $\mathrm{ZnO}^{15}$ and thus maintain the chemical integrity of the interface. Although $\mathrm{Tl}$ and $\mathrm{Pb}$ are undesirable due to their toxicity, Bi appears to be a promising dopant which could limit inter-diffusion and not significantly affect the strength of the interface. Evidence of an increased energy barrier for diffusion is given below.

\section{SILVER DIFFUSION IN DOPED ZINC OXIDE}

In a previous study, ${ }^{15}$ we proposed that doping $\mathrm{ZnO}$ with donor elements such as $\mathrm{Al}, \mathrm{Sc}$, and Sn can effectively increase the migration barrier for diffusion of $\mathrm{Ag}$ in $\mathrm{ZnO}$. However, these dopants also reduce the $\mathrm{Ag} / \mathrm{ZnO}$ interfacial adhesion as
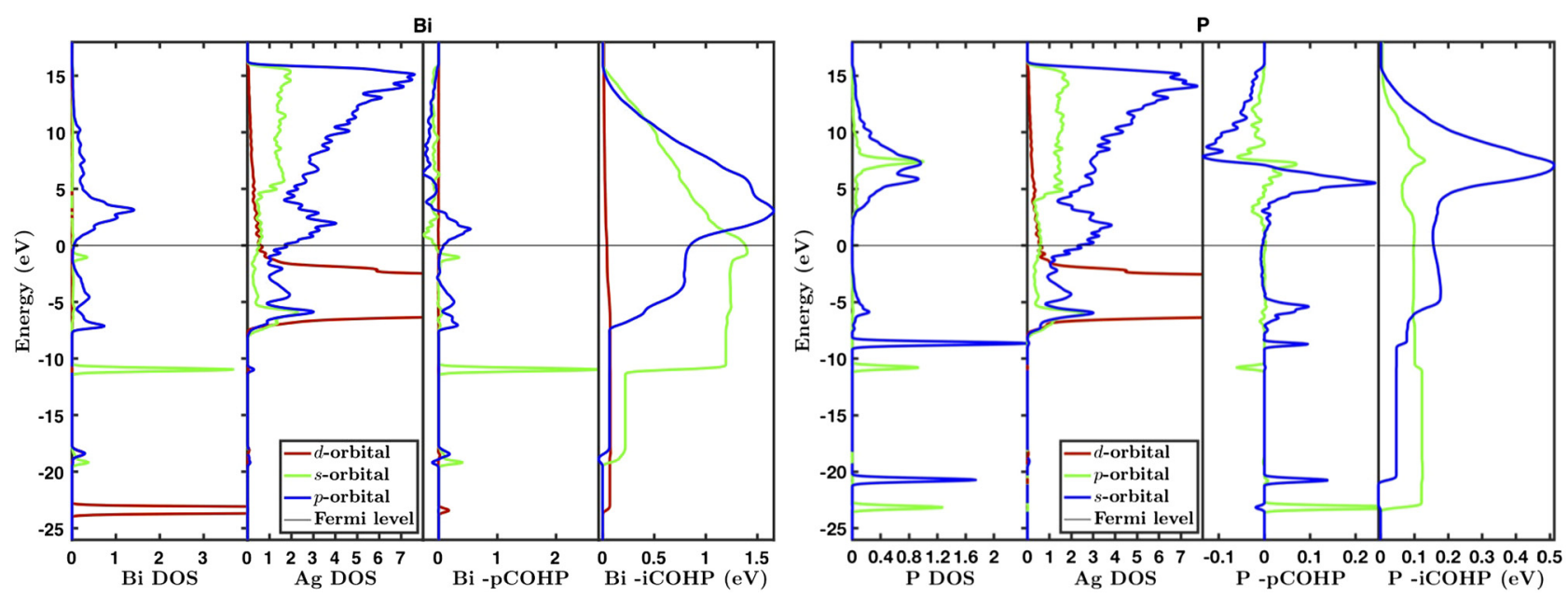

FIG. 6. Projected densities of states for a dopant (Bi or P) at the interface and for Ag atoms in the adjacent layer. Values of - pCOHP and -iCOHP are also shown. Positive contributions from - pCOHP are bonding interactions while negative ones are those of antibonding. 
the present work has shown. Monovalent elements such as $\mathrm{Li}$ appear to be a good choice in terms of interfacial strength and therefore it is of interest to study their kinetics in $\mathrm{ZnO}$. Nudged elastic band calculations have been performed on $\mathrm{Ag}$ diffusion in $\mathrm{Li}$-doped $\mathrm{ZnO}$ using the same computational setup as used previously. ${ }^{15}$ Unfortunately, $\mathrm{Li}$ is found to assist $\mathrm{Ag}$ diffusion involving a process in which the $\mathrm{Ag}$ atom substitutes for $\mathrm{Li}$ on a $\mathrm{Zn}$ site. This Li-assisted mechanism agrees well with experimental observations by Azarov et al. ${ }^{33}$ On the other hand, Bi appears to be a potentially useful dopant which may inhibit Ag diffusion because of its size and also not weaken the interface significantly. Similar nudged elastic band calculations have been performed on $\mathrm{Ag}$ diffusion in $\mathrm{Bi}$-doped $\mathrm{ZnO}$, and the energy barrier is found to be $1.35 \mathrm{eV}$ (see Fig. S3 in the supplementary material) which compares favorably with values of $0.75 \mathrm{eV}$ for pure $\mathrm{ZnO}$ and $0.89 \mathrm{eV}$ for Al-doped $\mathrm{ZnO} .{ }^{15}$ Thus, Bi does indeed inhibit Ag diffusion in $\mathrm{ZnO}$.

\section{v. CONCLUSIONS}

Using first principles DFT modeling, we have shown how substitutional doping can influence the mechanical strength of a $\mathrm{Ag} / \mathrm{ZnO}$ interface commonly found in various multilayer thinfilm systems. Two approaches have been used: calculation of the ideal work of separation and calculation of interfacial bond strengths using the iCOHP method. Both methods support the same general trend: replacing $\mathrm{Zn}$ with a monovalent dopant strengthens the interface while dopants with a valence greater than two weaken it. Isovalent dopants have little effect. The overall behavior can be explained largely in terms of a charge transfer mechanism at the interface. However, for a particular group of dopants which include $\mathrm{Tl}, \mathrm{Pb}$, and $\mathrm{Bi}$, small differences in the results obtained by the two methods reveal additional interactions that contribute to the interfacial strength. In particular, the iCOHP method indicates the presence of hybridization between the $s$ - and $p$-orbitals of the dopant and the orbitals of Ag just below the Fermi level. The presence of $d$-orbitals in the dopant enhances this hybridization by shifting the $s$ - and $p$-orbitals to higher energies. Although monovalent dopants appear to be preferred in terms of improving the strength of the interface, they do not inhibit inter-diffusion of $\mathrm{Ag}$ into the $\mathrm{ZnO}$ and therefore could lead to chemical degradation of the thinfilm system. Of the dopants studied in this work, Bi seems to be the best in that it does not significantly affect the interfacial strength and provides a relatively large barrier for $\mathrm{Ag}$ diffusion. It is clear, however, that complete control over the mechanics, kinetics, and chemistry of the interface requires a careful choice of dopant types and concentrations.

\section{SUPPLEMENTARY MATERIAL}

See supplementary material for (i) the planar electrostatic potential normal to the pristine and Bi-doped interface, (ii) densities of states and COHP for the pristine and doped interfaces, and (iii) minimum energy diffusion paths for $\mathrm{Ag}$ migration in $\mathrm{Bi}$ and $\mathrm{Li}$-doped $\mathrm{ZnO}$.

\section{ACKNOWLEDGMENTS}

T. Wang acknowledges the Cambridge Commonwealth, European and International Trust and the China Scholarship Council for financial support. The calculations were performed using the Cambridge University HPC Cluster as well as the UK National Supercomputing Service ARCHER. Access to the latter was provided by the UKCP and Materials Chemistry consortia which are both funded by the Engineering and Physical Sciences Research Consul (EPSRC) under Grant Nos. EP/P022596/1 and EP/L000202/1, respectively. The authors also thank Dr. Paul Warren from the Pilkington group and Professor Zheshuai Lin from the Chinese Academy of Sciences for useful discussions. In addition, we gratefully acknowledge the helpful advice provided by the LOBSTER developers. All the necessary computational data are included in the article or available at the Cambridge University Data Repository, https://doi.org/10.17863/CAM.25930.

${ }^{1}$ M. Arbab, MRS Bull. 22, 27 (1997).

${ }^{2}$ Z. Lin and P. D. Bristowe, Phys. Rev. B Condens. Matter Mater. Phys. 75, 205423 (2007).

${ }^{3}$ J. A. Ridealgh, MRS Proc. 890, 0890 (2005).

${ }^{4}$ R. G. Gordon, MRS Bull. 25, 52 (2000).

${ }^{5}$ D. S. Ginley and C. Bright, MRS Bull. 25, 15 (2000).

${ }^{6}$ C. G. Granqvist, Sol. Energy Mater. Sol. Cells 91, 1529 (2007).

${ }^{7}$ M. Arbab, Thin Solid Films 381, 15 (2001).

${ }^{8}$ N. Jedrecy, G. Renaud, R. Lazzari, and J. Jupille, Phys. Rev. B Condens. Matter Mater. Phys. 72, 045430 (2005).

${ }^{9}$ J. Yun, Adv. Funct. Mater. 27, 1606641 (2017).

${ }^{10}$ J. Chen, Z. Lin, S. J. Bull, C. L. Phillips, and P. D. Bristowe, J. Phys. D. Appl. Phys. 42, 214003 (2009).

${ }^{11}$ Z. Lin and P. D. Bristowe, J. Appl. Phys. 106, 013520 (2009).

${ }^{12}$ E. Ando and M. Miyazaki, Thin Solid Films 392, 289 (2001).

${ }^{13}$ M. Miyazaki and E. Ando, J. Non Cryst. Solids 178, 245 (1994).

${ }^{14}$ E. Ando, S. Suzuki, N. Aomine, M. Miyazaki, and M. Tada, Vacuum 59, 792 (2000).

${ }^{15}$ T. Wang and P. D. Bristowe, Acta Mater. 137, 115 (2017).

${ }^{16}$ R. D. Shannon, Acta Crystallogr. Sect. A 32, 751 (1976).

${ }^{17}$ R. Sharma, K. Sehrawat, and R. M. Mehra, Curr. Appl. Phys. 10, 164 (2010).

${ }^{18}$ J. H. Lee and B. O. Park, Thin Solid Films 426, 94 (2003).

${ }^{19}$ G. Kresse and J. Hafner, Phys. Rev. B 47, 558 (1993).

${ }^{20}$ G. Kresse and J. Furthmüller, Phys. Rev. B 54, 11169 (1996).

${ }^{21}$ J. P. Perdew, K. Burke, and M. Ernzerhof, Phys. Rev. Lett. 77, 3865 (1996).

${ }^{22}$ J. P. Perdew, A. Ruzsinszky, G. I. Csonka, O. A. Vydrov, G. E. Scuseria, L. A. Constantin, X. Zhou, and K. Burke, Phys. Rev. Lett. 100, 136406 (2008).

${ }^{23}$ P. E. Blöchl, Phys. Rev. B 50, 17953 (1994).

${ }^{24}$ G. Kresse, Phys. Rev. B 59, 1758 (1999).

${ }^{25}$ R. Dronskowski and P. E. Bloechl, J. Phys. Chem. 97, 8617 (1993).

${ }^{26}$ V. L. Deringer, A. L. Tchougréeff, and R. Dronskowski, J. Phys. Chem. A 115, 5461 (2011).

${ }^{27}$ S. Maintz, V. L. Deringer, A. L. Tchougréeff, and R. Dronskowski, J. Comput. Chem. 34, 2557 (2013).

${ }^{28}$ S. Maintz, V. L. Deringer, A. L. Tchougréeff, and R. Dronskowski, J. Comput. Chem. 37, 1030 (2016).

${ }^{29}$ K. Momma and F. Izumi, J. Appl. Crystallogr. 44, 1272 (2011).

${ }^{30}$ S. C. Abrahams and J. L. Bernstein, Acta Crystallogr. Sect. B Struct. Crystallogr. Cryst. Chem. 25, 1233 (1969).

${ }^{31}$ K. Yim, J. Lee, D. Lee, M. Lee, E. Cho, H. S. Lee, H.-H. Nahm, and S. Han, Sci. Rep. 7, 40907 (2017).

${ }^{32}$ A. Smakula and J. Kalnajs, Phys. Rev. 99, 1737 (1955).

${ }^{33}$ A. Azarov, L. Vines, P. Rauwel, E. Monakhov, and B. G. Svensson, J. Appl. Phys. 119, 185705 (2016). 